\title{
Adubação fosfatada no sulco e foliar afetando a produtividade de grãos do feijoeiro comum
}

\section{Phosphorus fertilization in the sowing furrow and foliar as affecting the common beans grain yield}

\author{
Adriano Stephan Nascente ${ }^{1 *}$; Tarcísio Cobucci ${ }^{1}$; \\ Djalma Martinhão Gomes de Sousa²; Daniel de Paiva Lima
}

\begin{abstract}
Resumo
O fósforo $(\mathrm{P})$ é um elemento essencial no metabolismo das plantas. Devido à elevada taxa de fixação do $\mathrm{P}$ em solos tropicais, o aproveitamento pela cultura varia de $5 \%$ a $25 \%$. Uma opção seria fornecê-lo por meio da adubação foliar, favorecendo a redução da quantidade de adubos fosfatados aplicados via solo, gerando ganhos econômicos e ambientais. Entretanto, essa prática ainda é realizada sem embasamento científico, o que dificulta a obtenção de resultados confiáveis. O objetivo deste trabalho foi testar a hipótese de que a aplicação de $\mathrm{P}$ foliar aumenta a eficiência de utilização de $\mathrm{P}$ do solo pelo feijoeiro. $\mathrm{O}$ experimento foi realizado por três anos agrícolas consecutivos em solo representativo do Cerrado, em cultivo de inverno com irrigação suplementar. O delineamento experimental utilizado foi o de blocos ao acaso, com quatro repetições, em esquema fatorial 4 (doses de P no solo) x 4 (doses de P via foliar). $\mathrm{O}$ aumento das doses de $\mathrm{P}$ aplicadas via solo e foliar proporcionaram aumento do número vagens $\mathrm{m}^{-2}$, produtividade e teor de $\mathrm{P}$ na planta do feijoeiro comum. A maior produtividade do feijoeiro $(3446 \mathrm{~kg}$

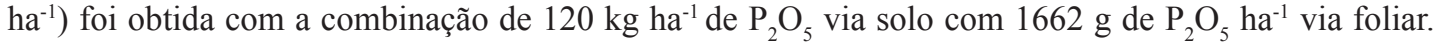
A resposta da cultura à aplicação de $\mathrm{P}$ no solo é incrementada com a aplicação do nutriente via foliar.

Palavras-chave: Phaseolus vulgaris, nutrição mineral de plantas, fósforo, cerrado
\end{abstract}

\begin{abstract}
Phosphorus $(\mathrm{P})$ is an essential element in plant metabolism, and limiting the production of common beans. One option would be through foliar fertilization, favoring the reduction of phosphate fertilizers in the soil, causing economic and environmental gains. However, this practice is still carried out without scientific basis, making it difficult to obtain reliable results. The aim of this study was to test the hypothesis that the foliar $\mathrm{P}$ application increases the efficiency of utilization of $\mathrm{P}$ from soil by common beans. The trial was conducted for three consecutive years in a representative soil of the Cerrado in the winter season with supplemental irrigation. The experimental design was randomized blocks with four replicates in a factorial scheme 4 (rates of P applied in the soil) $\mathrm{x} 4$ (rates of $\mathrm{P}$ applied foliar). The increased in the rates of $\mathrm{P}$ applied to soil and foliar provided increased in the number pods $\mathrm{m}^{-2}$, grain yield and $\mathrm{P}$ content in the common bean plant. The highest grain yield $\left(3,446 \mathrm{~kg} \mathrm{ha}^{-1}\right)$ was obtained with

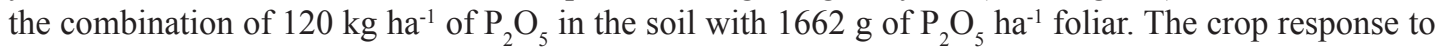
the application of $\mathrm{P}$ in the soil was increased with foliar application.
\end{abstract}

Key words: Phaseolus vulgaris, mineral nutrition of plants, phosphorus, cerrado

\footnotetext{
${ }^{1}$ Eng $^{\mathrm{os}} \mathrm{Agr}^{\mathrm{os}}$, Drs. Pesquisadores, Embrapa Arroz e Feijão, Santo Antônio de Goiás, GO. E-mail: adriano.nascente@embrapa.br; tarcisio.cobucci@embrapa.br

${ }^{2}$ Eng $^{\mathrm{O}} \mathrm{Agr}{ }^{\mathrm{O}}$, Pesquisador, Dr., Embrapa Cerrados, Planaltina, DF. E-mail: djalma.sousa@embrapa.br

${ }^{3}$ Eng $^{\circ}$ Agr $^{\circ}$, Discente do Curso de Mestrado em Agronomia, Embrapa Arroz e Feijão, Santo Antônio de Goiás, GO. E-mail: daniel_apl@hotmail.com

* Autor para correspondência
} 
A cultura do feijão possui grande importância econômica ao Brasil, uma vez que, a área cultivada, na safra 2010/2011 foi de cerca de 4,01 milhões de hectares, o que correspondeu a produção de 3,8 milhões de toneladas (CONAB, 2013). Entretanto, apesar da sua importância, ainda é constatada baixa utilização de tecnologia, principalmente na primeira e na segunda safra, resultando em baixa produtividade, equivalente a $945 \mathrm{~kg} \mathrm{ha}^{-1}$. A terceira safra, caracterizada como mais tecnificada, teve média de produtividade de $3.607 \mathrm{~kg} \mathrm{ha}^{-1}$ na safra 2010/2011, indicando que há retorno em produtividade de grãos em razão do uso de tecnologia.

Entre os aspectos que devem ser melhorados na cultura, está o manejo da adubação das plantas, uma vez que com o fornecimento adequado e equilibrado de nutrientes para o feijoeiro, pelo uso de fertilizantes, pode-se obter aumentos significativos na produtividade da cultura (MIRANDA et al., 2000; ANDRADE et al., 2004; ZUCARELI et al., 2010; ARF et al., 2011; MELÉM JÚNIOR et al., 2011). Neste sentido, tem-se o P, elemento essencial no metabolismo das plantas, no qual se constata que a resposta do feijoeiro à utilização do $\mathrm{P}$ via solo é bem definida, contribuindo de forma significativa para o aumento da produtividade de grãos, do desenvolvimento radicular, além de favorecer o aumento do número de vagens e da massa de grãos (PELÁ et al., 2009; ZUCARELI et al., 2010). Assim, verifica-se que esse elemento é um dos mais limitantes na produção do feijoeiro, principalmente em solos de baixa fertilidade como os de Cerrado (FAGERIA; BALIGAR, 1996; FAGERIA; SANTOS; MOREIRA, 2010). A necessidade em $\mathrm{P}$ requerida pelas plantas do feijoeiro é menor que a de potássio $(\mathrm{K})$ e nitrogênio $(\mathrm{N})$, no entanto, a quantidade aplicada normalmente é superior (VIEIRA, 2006). Isso ocorre devido à elevada taxa de fixação do $\mathrm{P}$ em solos tropicais, causada principalmente por precipitação por $\mathrm{Fe}$ e Al, reação com óxidos hidratados dos mesmos metais e reação com argilas silicatadas, devido a isso o aproveitamento pela cultura varia de $5 \%$ a $25 \%$ (MALAVOLTA, 1980). Uma opção seria fornecêlo por meio da adubação foliar, proporcionando redução da quantidade de adubos fosfatados aplicados via solo, gerando ganhos econômicos e ambientais, uma vez que esses são produzidos a partir de reservas minerais de caráter não renovável (PELÁ et al., 2009).

A adubação foliar se justifica por ser o $\mathrm{P}$ elemento móvel, que pode ser redistribuído do local aplicado para outras partes da planta (SILVA, 2006). Além disso, do início do estádio reprodutivo até a maturação a atividade radicular e a absorção do nutriente diminuem, por outro lado, há grande translocação do $\mathrm{P}$ das folhas para as sementes em formação. Neste sentido, a adubação foliar poderia repor os nutrientes nas folhas, mantendo a taxa de fotossíntese por mais tempo, com possíveis reflexos na produtividade da cultura (BORKERT, 1987). Adicionalmente, a aplicação do $\mathrm{P}$ foliar na fase vegetativa, pode proporcionar aumento da absorção de nutrientes do solo (ROSOLEM; BOARETO, 1987). Essa hipótese foi confirmada na cultura da soja por Humbert (1983) na qual a aplicação foliar de NPK durante o período vegetativo proporcionou aumentos nas quantidades dos nutrientes nas plantas, as quais eram superiores às quantidades aplicadas via solo, permitindo inferir o efeito estimulante da adubação foliar na absorção radicular.

Em diversos trabalhos de pesquisa foram relatados aumentos de produtividade das culturas devido à aplicações foliares de P (PRIMAVESI, 1981; MURAOKA; NEPTUNE, 1981; HAQ; MALLARINO, 2000; PELÁ et al., 2003; REZENDE et al., 2005; KUBOTA et al., 2008; PELÁ et al., 2009). O uso da adubação foliar com $\mathrm{P}$ vem sendo incentivado aos agricultores sem embasamento científico, apresentando resultados controversos e sem confiabilidade, não sendo ainda prática corriqueira na agricultura (ROSOLEM; BOARETTO, 1987; ALVARENGA et al., 2000; HAQ; MALLARINO, 2000; PELÁ et al., 2009). Assim partindo-se da hipótese de que a aplicação 
de $\mathrm{P}$ foliar aumenta a eficiência de utilização de $\mathrm{P}$ do solo pelo feijoeiro proporcionando diminuição na aplicação do P via solo.

O objetivo desse trabalho foi de avaliar o efeito de doses de $\mathrm{P}$ aplicados via solo e via foliar nos componentes de produção, produtividade de grãos, teor de $\mathrm{P}$ nas plantas e taxa de recuperação de $\mathrm{P}$ pelo feijoeiro.

O experimento foi desenvolvido na Embrapa Arroz e Feijão, localizada no município de Santo Antônio de Goiás, GO (16 $26^{\circ}$ 'de latitude, $49^{\circ}$ $17^{\prime}$ de longitude e $823 \mathrm{~m}$ de altitude) durante três anos consecutivos 2009, 2010 e 2011. O clima é classificado como Aw, tropical de savana, mesotérmico, segundo a classificação de Koppen. A média histórica da região (1983-2010) é de 32, 8,3, 4,3 e 12,6 mm precipitado nos meses de maio, junho, julho e agosto, respectivamente e temperaturas médias de $21,8,20,6,20,8$ e $22,6^{\circ} \mathrm{C}$ nos mesmos meses, respectivamente (SILVA et al., 2010).

O solo é classificado como Latossolo Vermelho distrófico, de textura argilosa (EMBRAPA, 1999), com relevo suavemente ondulado. Antes da instalação do experimento realizou-se a caracterização química, granulométrica e densidade do solo na profundidade: $0-20 \mathrm{~cm}$ (Tabela 1), segundo a metodologia proposta no manual de métodos da Embrapa (CLAESSEN, 1997). O experimento foi instalado em área de plantio direto sobre palhada de Urochroa brizantha.

Tabela 1. Atributos químicos das áreas onde foram conduzidos os ensaios. Fazenda Capivara 2009,2010 e 2011.

\begin{tabular}{|c|c|c|c|c|c|c|c|c|c|c|c|c|}
\hline Ano & $\mathrm{pH}$ & MO & $\mathrm{K}$ & $\mathrm{Ca}$ & $\mathrm{Mg}$ & $\mathrm{Al}$ & $\mathrm{P}$ & B & $\mathrm{Cu}$ & $\mathrm{Fe}$ & $\mathrm{Mn}$ & $\mathrm{Zn}$ \\
\hline & (água) & $\mathrm{g} \mathrm{dm}^{-3}$ & \multicolumn{5}{|c|}{$------\mathrm{cmol}_{\mathrm{c}} \mathrm{dm}^{-3}------$} & \multicolumn{5}{|c|}{----------------'mg dm³-'----------------- } \\
\hline 2009 & 4,5 & 17 & 0,33 & 1,7 & 0,8 & 0,1 & 22,2 & 0,24 & 1,5 & 32,5 & 11,6 & 4,2 \\
\hline 2010 & 4,3 & 18 & 0,4 & 1,5 & 0,7 & 0,2 & 16,0 & 0,23 & 1,4 & 33,4 & 10,7 & 6,5 \\
\hline 2011 & 4,7 & 19 & 0,35 & 2,0 & 0,7 & 0,1 & 18,3 & 0,30 & 1,2 & 32,5 & 11,5 & 6,4 \\
\hline
\end{tabular}

$\mathrm{pH}$ - potencial hidrogeniônico, $\mathrm{MO}$ - matéria orgânica, $\mathrm{K}$ - potássio, $\mathrm{Ca}$ - cálcio, $\mathrm{Mg}$ - magnésio, $\mathrm{Al}$ - alumínio, $\mathrm{P}$ - fósforo, $\mathrm{B}$ boro, Fe - ferro, Mn - manganês, Zn - zinco.

Fonte: Elaborado pelos autores.

O delineamento experimental utilizado foi em blocos ao acaso, em esquema fatorial 4 x 4, com quatro repetições. Os tratamentos foram constituídos por quatro doses de $\mathrm{P}$ aplicados via solo $(0 ; 40 ; 80$ e $120 \mathrm{~kg} \mathrm{ha}^{-1}$ de $\mathrm{P}_{2} \mathrm{O}_{5}$ ) e quatro doses de $\mathrm{P}$ aplicadas via foliar (zero; 831,3; $1.662,6$ e 2.493,9 $\mathrm{g} \mathrm{P}_{2} \mathrm{O}_{5}$ ha$\left.{ }^{1}\right)$. As dimensões das parcelas foram de $4,5 \mathrm{~m}$ de largura e $6 \mathrm{~m}$ de comprimento, perfazendo $27 \mathrm{~m}^{2}$.

A semeadura do feijoeiro comum, cultivar Pérola, foi realizada nos dias 15/05/2009, 19/05/2010 e 23/05/2011. O espaçamento entre as linhas foi de $0,50 \mathrm{~m}$ e a densidade de semeadura foi de 15 sementes por metro linear. Os tratos culturais foram realizados de acordo com as necessidades da cultura, utilizando-se os produtos recomendados. Utilizou-se sistema fixo de irrigação convencional por aspersão com vazão de $3,3 \mathrm{~mm} \mathrm{~h}^{-1}$ nos aspersores e o cálculo da lâmina d'água foi realizado segundo as recomendações de Doorenbos e Pruitt (1976).

A aplicação de $\mathrm{P}$ via solo foi realizada juntamente com a aplicação de $\mathrm{N}$ e $\mathrm{K}$ durante a semeadura do feijoeiro comum. O P foliar foi fornecido pelo produto comercial P51, que contém $1 \%$ de $\mathrm{N}$ e $51 \% \mathrm{P}_{2} \mathrm{O}_{5}$, com densidade de $1,63 \mathrm{~kg}$ $\mathrm{L}^{-1}$, sendo as doses para cada tratamento divididas em duas aplicações. A primeira aplicação ocorreu quando o feijoeiro apresentava três trifólios e a segunda, com seis trifólios. Para a aplicação foliar foi utilizado pulverizador manual tipo costal com pressão constante de $\mathrm{CO}_{2}$, utilizando-se bico cônico tipo TX-VS2, com volume de calda aproximado de $100 \mathrm{~L} \mathrm{ha}^{-1}$. 
Para a avaliação dos componentes de produção (número de vagens planta ${ }^{-1}$, número de grãos vagem ${ }^{-1}$ e massa de 100 grãos) foram coletadas 10 plantas ao acaso em cada parcela. Avaliou-se a produtividade de grãos (130 $\mathrm{g} \mathrm{kg}^{-1}$ de umidade) realizando-se a colheita de três linhas centrais de cinco metros em cada parcela desprezando-se $0,5 \mathrm{~m}$ de cada lado. Também se determinou o teor de P na planta toda (grãos + folhas + caule + vagens + raízes), por meio de análise química, conforme descrito em Claessen (1997).

Os dados foram submetidos à análise de variância (ANOVA), teste F, em esquema fatorial 4 (doses $\mathrm{P}$ via solo) x 4 (doses de $\mathrm{P}$ via foliar) x 3 (anos de condução dos experimentos) e quando o teste $\mathrm{F}$ foi significativo, procedeu-se ao teste Tukey $(\mathrm{p} \leq 0,05)$.

Para o número de vagens $\mathrm{m}^{-2}$, houve efeitos isolados do P aplicado via solo e foliar (Tabela 2). Com relação às doses aplicadas via solo, os dados foram ajustados à equação polinomial de segundo grau, onde constatou-se valor máximo estimado foi na dose de $70 \mathrm{~kg} \mathrm{ha}^{-1}$ (Figura 1a). Quanto à aplicação via foliar, também os dados foram ajustados à equação polinomial, e os maiores valores estimado foram obtidos na aplicação de $1.662 \mathrm{~g} \mathrm{P}_{2} \mathrm{O}_{5} \mathrm{ha}^{-1}$ (Figura 1b). Nesse sentido, verificou-se resposta à aplicação de $\mathrm{P}$ para esse componente da produção do feijoeiro até determinadas doses, não tendo portanto, ajustes lineares. Estes resultados contradizem os obtidos por Valderrama et al. (2009) que não constataram incremento no número de vagens para as doses de $\mathrm{P}$ utilizadas ( 0 a $150 \mathrm{~kg} \mathrm{ha}^{-1}$ de $\mathrm{P}_{2} \mathrm{O}_{5}$ ). Eles explicaram o resultado como sendo efeito da baixa exigência da cultura nesse nutriente, o que também foi descrito por Rosolem e Boaretto (1987). No entanto corroboram os resultados descritos por Zucareli et al. (2006), Pelá et al. (2009) e Zucareli et al. (2011) em relação ao aumento das doses de P aplicadas no solo. Fageria, Barbosa Filho e Stone (2004) e Zucareli et al. (2006) verificaram que o número de vagens é o componente de produção que mais influencia no aumento da produtividade do feijoeiro, e é diretamente influenciado pelas condições do meio, entre elas a adubação com P via solo ou foliar.

O aumento dos teores no solo ou foliares não influenciaram o número grãos vagem ${ }^{-1}$ e massa de 100 grãos (Tabela 2). O número de grãos vagem ${ }^{-1}$ é característica peculiar de cada cultivar, determinada geneticamente, tendo pouca influência do meio (PELÁ et al., 2009), entretanto a massa de 100 grãos é mais influenciada pelas condições do meio, mas não foi alterada pelas doses de P. Corroborando essas informações, Zucareli et al. (2006), Pelá et al. (2009), Valderrama et al. (2009), Melém Júnior et al. (2011) e Zucareli et al. (2011) também não encontraram diferenças significativas com o incremento das doses de $\mathrm{P}$ via solo sobre o número de grãos vagens ${ }^{-1}$ e massa de 100 grãos.

Para a produtividade de grãos, verificou-se efeitos isolado de ano e efeito da interação entre o $\mathrm{P}$ aplicado no solo e o aplicado via foliar (Tabela 2). Dessa forma, constatou-se maiores produtividades no ano 2011 (3.279 $\mathrm{kg} \mathrm{ha}^{-1}$ ), o que pode ter sido reflexo do maior massa de 100 grãos obtido nessa safra. Assim, no ano 2010, observou-se a menor produtividade e teve-se a menor massa 100 grãos (24,8 gramas).

Com relação à interação entre as formas de aplicação de $\mathrm{P}$, os dados foram ajustados a equações lineares (Figura 2a). Além disso, verificou-se que quando não se aplicou $\mathrm{P}$ no solo o incremento na produtividade do feijoeiro com a aplicação de $\mathrm{P}$ via foliar foi bem maior, quando comparado com a aplicação de $120 \mathrm{~kg}$ de $\mathrm{P}$ no solo (Figura 2a), saindo no primeiro caso de $2.320 \mathrm{~kg} \mathrm{ha}^{-1}\left(0 \mathrm{~g} \mathrm{P}_{2} \mathrm{O}_{5} \mathrm{ha}^{-1}\right)$ para $2.818 \mathrm{~kg} \mathrm{ha}^{-1}\left(1.662 \mathrm{~g} \mathrm{P}_{2} \mathrm{O}_{5} \mathrm{ha}^{-1}\right)$, aumento de mais de $18 \%$. Na aplicação de $120 \mathrm{~kg}$ de $\mathrm{P}_{2} \mathrm{O}_{5}$ ha $^{-1}$ houve incremento de $3.075 \mathrm{~kg} \mathrm{ha}^{-1}\left(0 \mathrm{~g} \mathrm{P}_{2} \mathrm{O}_{5}\right.$ ha $\left.^{-1}\right)$ para $3.446 \mathrm{~kg} \mathrm{ha}^{-1}$ (1662 $\mathrm{g} \mathrm{P}_{2} \mathrm{O}_{5} \mathrm{ha}^{-1}$ ) na produtividade do feijoeiro, ou seja, aumentou de 10,8\%. Corroborando essas informações, Pelá et al. (2009) também obtiveram aumentos significativos na produtividade do feijoeiro quando o suprimento de $P$ foi via foliar em comparação com a não aplicação 
do P no solo. Vale destacar que ocorreu a maior produtividade do feijoeiro com a aplicação da dose de $1.662 \mathrm{~g} \mathrm{P}_{2} \mathrm{O}_{5}$ ha $^{-1}$ via foliar e não na dose máxima. Além disso, a aplicação conjunta do P no solo com o foliar proporcionou maiores produtividades, provavelmente, porque o $\mathrm{P}$ aplicado via solo foi responsável pelo maior desenvolvimento do sistema radicular (FAGERIA; BARBOSA FILHO; STONE, 2004). Enquanto no início do estádio reprodutivo até a maturação, a atividade radicular e a absorção do nutriente diminuem. Nesse sentido, apesar de não ter sido avaliado nesse experimento, existe a hipótese de que pode haver também grande translocação do $\mathrm{P}$ das folhas para as sementes em formação. Assim, a adubação foliar pode ter proporcionado a reposição dos nutrientes nas folhas, mantendo a taxa de fotossíntese por mais tempo, e isso se traduziu em maiores produtividade, relatos semelhantes foram descritos por Borkert (1987). Adicionalmente, verificou-se que a combinação das doses que proporcionou a maior produtividade de grãos do feijoeiro (3.446 $\mathrm{kg} \mathrm{ha}^{-1}$ ) foi $120 \mathrm{~kg} \mathrm{P}_{2} \mathrm{O}_{5}$ $\mathrm{ha}^{-1}$ via solo e $1.662 \mathrm{~g} \mathrm{P}_{2} \mathrm{O}_{5} \mathrm{ha}^{-1}$ via foliar.

Tabela 2. Componentes de produção, produtividade e teor de fósforo $(\mathrm{P})$ nas plantas de feijão em função do ano, $\mathrm{P}$ aplicado no solo (P solo) e P aplicado via foliar (P foliar). Santo Antônio de Goiás, 2009, 2010 e 2011.

\begin{tabular}{lccccc}
\hline \multicolumn{1}{c}{ Fatores } & Vagens $\mathrm{m}^{-2}$ & Grãos vagem $^{-1}$ & Massa 100 grãos & Produtividade & Teor de P \\
\hline \multicolumn{1}{c}{$\underline{\text { Ano }}$} & número & número & gramas & $\mathrm{kg} \mathrm{ha}^{-1}$ & $\mathrm{~g} \mathrm{~kg}^{-1}$ \\
2009 & $260 \mathrm{a}$ & $4,4 \mathrm{a}$ & $27,3 \mathrm{ab}$ & $3001 \mathrm{~b}$ & $18,6 \mathrm{a}$ \\
2010 & $270 \mathrm{a}$ & $4,9 \mathrm{a}$ & $24,8 \mathrm{~b}$ & $2705 \mathrm{c}$ & $18,1 \mathrm{a}$ \\
$\quad$ P solo $\left(\mathrm{kg} \mathrm{ha}^{-1}\right)$ & $265 \mathrm{a}$ & $4,8 \mathrm{a}$ & $29,7 \mathrm{a}$ & $3279 \mathrm{a}$ & $18,7 \mathrm{a}$ \\
0 & & & & & \\
40 & 238 & 4,4 & 26,4 & 2707 & 16,7 \\
80 & 276 & 4,3 & 27,2 & 2910 & 16,8 \\
120 & 271 & 4,4 & 28,0 & 3007 & 19,3 \\
$\quad$ P foliar g $\left(\mathrm{ha}^{-1}\right)$ & 254 & 4,5 & 27,5 & 3356 & 21,7 \\
0 & & & & \\
831 & 241 & 4,3 & 27,5 & 2764 & 16,3 \\
1662 & 259 & 4,5 & 27,0 & 2930 & 19,4 \\
2493 & 276 & 4,3 & 27,0 & 3226 & 19,9 \\
Fatores & 261 & 4,4 & 27,6 & 3061 & 19,0 \\
Ano (A) & & ANAVA (Probabilidade do teste F) & & \\
P solo (PS) & 0,1209 & 0,2001 & 0,0451 & $<0,001$ & 0,1982 \\
P foliar (PF) & $<0,001$ & 0,3397 & 0,0983 & $<0,001$ & 0,0436 \\
A x PS & $<0,001$ & 0,5102 & 0,3839 & $<0,001$ & 0,0441 \\
A x PF & 0,0765 & 0,3677 & 0,2257 & 0,2359 & 0,2355 \\
PF x PS & 0,0654 & 0,4813 & 0,1875 & 0,2314 & 0,1419 \\
A x PS x PF & 0,1123 & 0,3954 & 0,4427 & 0,0437 & 0,1786 \\
\hline
\end{tabular}

${ }^{1}$ Médias seguidas de mesma letra, na coluna, não diferem entre si pelo teste Tukey, $\mathrm{p} \leq 0,05$.

Fonte: Elaborado pelos autores. 
Figura 1. Número de vagens $\mathrm{m}^{-2}$ de plantas de feijoeiro comum em função da quantidade de $\mathrm{P}_{2} \mathrm{O}_{5}$ aplicada via solo (a) e via foliar (b), Santo Antônio de Goiás, 2009, 2010 e 2011. ** - significativo para p $\leq 0,01$.
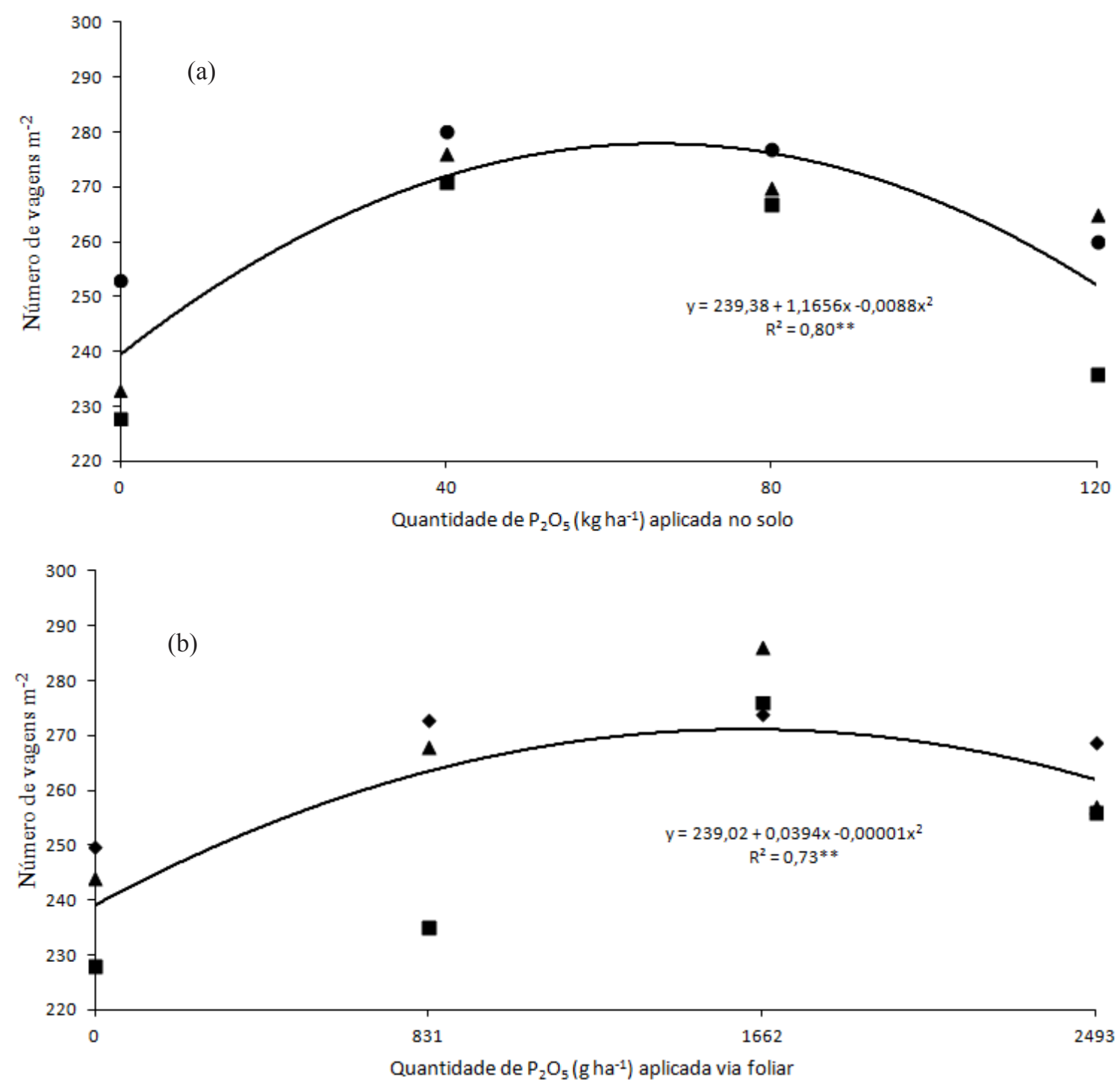

Fonte: Elaborado pelos autores.

O mesmo se verificou com o efeito da aplicação de P no solo dentro da aplicação de $\mathrm{P}$ foliar (Figura 2b). Ocorreu incremento de 2.230 ( 0 de $\mathrm{P}$ via solo) para $3.013 \mathrm{~kg} \mathrm{ha}^{-1}\left(120 \mathrm{~kg} \mathrm{P}_{2} \mathrm{O}_{5} \mathrm{ha}^{-1}\right)$, aumento de $26 \%$ quando não se aplicou $\mathrm{P}$ via foliar. Com aplicação de $2.493 \mathrm{~g} \mathrm{P}_{2} \mathrm{O}_{5} \mathrm{ha}^{-1}$ via foliar o incremento na produtividade do feijoeiro foi de $2.683 \mathrm{~kg} \mathrm{ha}^{-1}$ (0 de $\mathrm{P}$ via solo) para $2.981 \mathrm{~kg} \mathrm{ha}^{-1}\left(120 \mathrm{~kg} \mathrm{P}_{2} \mathrm{O}_{5}\right.$ $\left.\mathrm{ha}^{-1}\right)$. Esses resultados permitem inferir que houve aumento da produtividade com o incremento das doses de P no solo, e essa foi ainda maior quando se fez a adubação foliar, corroborando os relatos de Silberstein e Wittwer (1951); Dixon (2003); Girma et al. (2007); Noack, McBeath e McLaughlin (2010). No entanto, o incremento na produtividade é menor, quando se aumenta as doses de $\mathrm{P}$ aplicadas via solo, possivelmente, devido ao aumento da fixação do P no solo, o que resultaria em redução da disponibilidade do nutriente para a planta. 
Figura 2. Variação da produtividade do feijoeiro comum em função das doses crescentes de P aplicadas no solo (a) e via foliar (b). Santo Antônio de Goiás, 2009, 2010 e 2011. * - significativo para $p \leq 0,05$. ** - significativo para $p<0,01$.
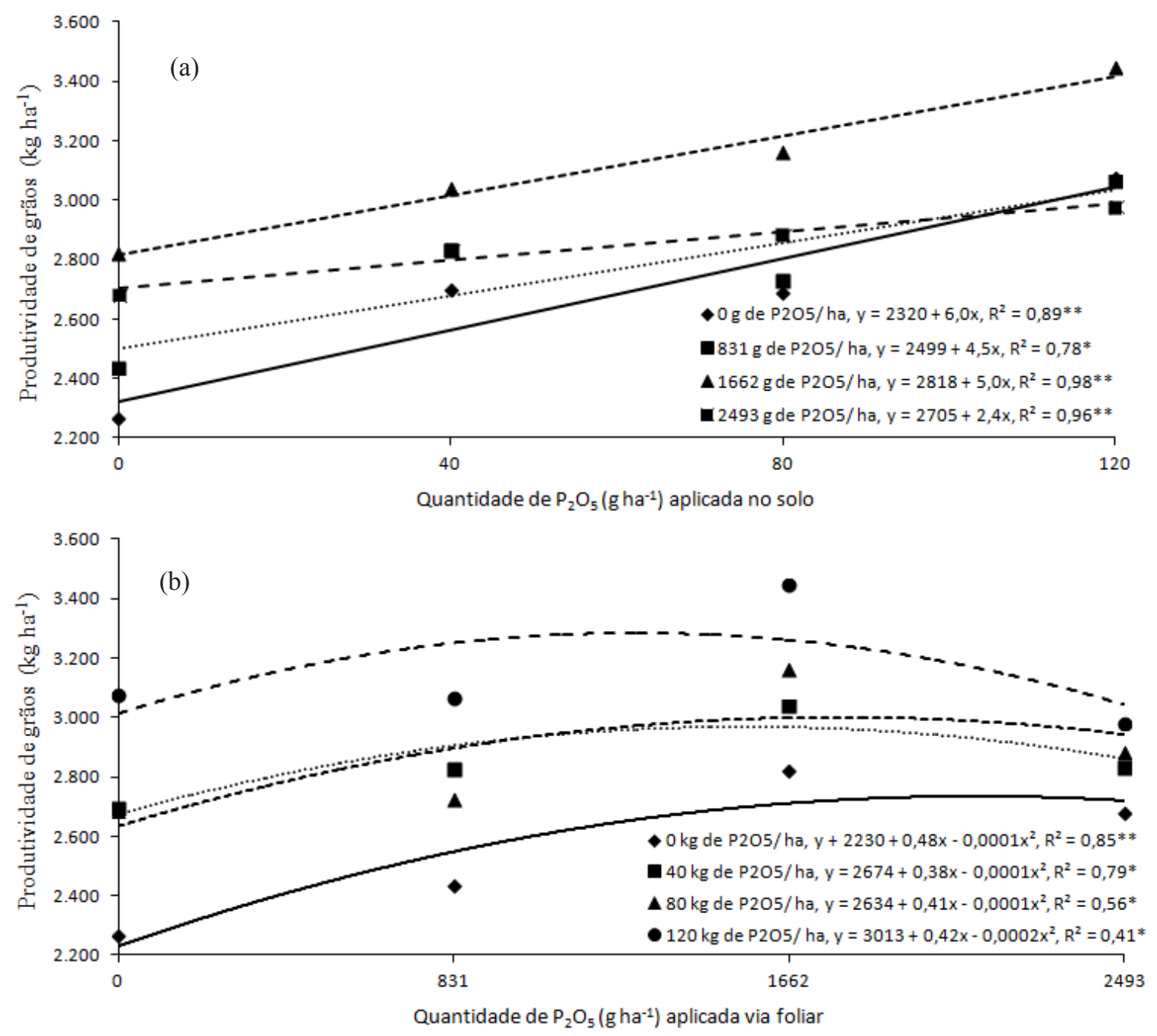

Fonte: Elaborado pelos autores.

Segundo Silva (2006) e Pelá et al. (2009), uma forma de reduzir as perdas por fixação do P no solo seria a aplicação conjunta desse nutriente via solo e via foliar, o que traria benefícios econômicos devido a redução da quantidade de $\mathrm{P}$ aplicada no solo e ambientais, pois o P é produzido pela extração de jazidas minerais de caráter não renovável. No presente trabalho foi observado que a aplicação foliar reduziu a necessidade de $\mathrm{P}$ via solo, o que corrobora as informações desses dois autores. Além disso, é importante ressaltar, que as aplicações de $\mathrm{P}$ via solo e foliares proporcionaram aumento da produtividade do feijoeiro (Figura 1). Resultados semelhantes foram obtidos por Rezende et al. (2005), na soja, e por Muraoka e Neptune (1981), Pelá et al. (2003), Pelá et al. (2009) e Melém Júnior et al. (2011), com feijoeiro. Segundo Fageria, Santos e Moreira (2010) e Zucareli et al. (2010, 2011), o P é um dos principais limitantes à produtividade das culturas e necessita de adequado manejo para obter produtividades desejáveis.

$\mathrm{O}$ aumento dos teores de $\mathrm{P}$ aplicados via solo teve efeito linear para o acúmulo desse nutriente 
nas plantas (Figura 3a). Com relação à aplicação via foliar, os dados foram ajustados à equação polinomial de segundo grau, com valores crescentes até próximo de $1.662 \mathrm{~g}$ de $\mathrm{P}_{2} \mathrm{O}_{5} \mathrm{ha}^{-1}$ (Figura 3b). Segundo Fageria, Barbosa Filho e Stone (2004) existe correlação significativa e positiva entre a acumulação de $\mathrm{P}$ nas plantas de feijoeiro e a produtividade da cultura, com isso, é de se esperar que, como ocorreu neste trabalho, o aumento do acúmulo de $\mathrm{P}$ pelas plantas proporcionou a maior produtividade do feijoeiro. De acordo com Zucareli et al. (2006), o P deve ser fornecido à planta de forma contínua, uma vez que a sua absorção máxima ocorre durante a pré-floração, e seu acúmulo nos grãos ocorre principalmente devido à redistribuição do $\mathrm{P}$ contido nas folhas e caules.

Figura 3. Variação do teor de $\mathrm{P}$ nas plantas de feijoeiro comum em função da quantidade de $\mathrm{P}_{2} \mathrm{O}_{5}$ aplicada via solo (a) e via foliar (b). Santo Antônio de Goiás, 2009, 2010 e 2011. ** - significativo para p $\leq 0,01$.
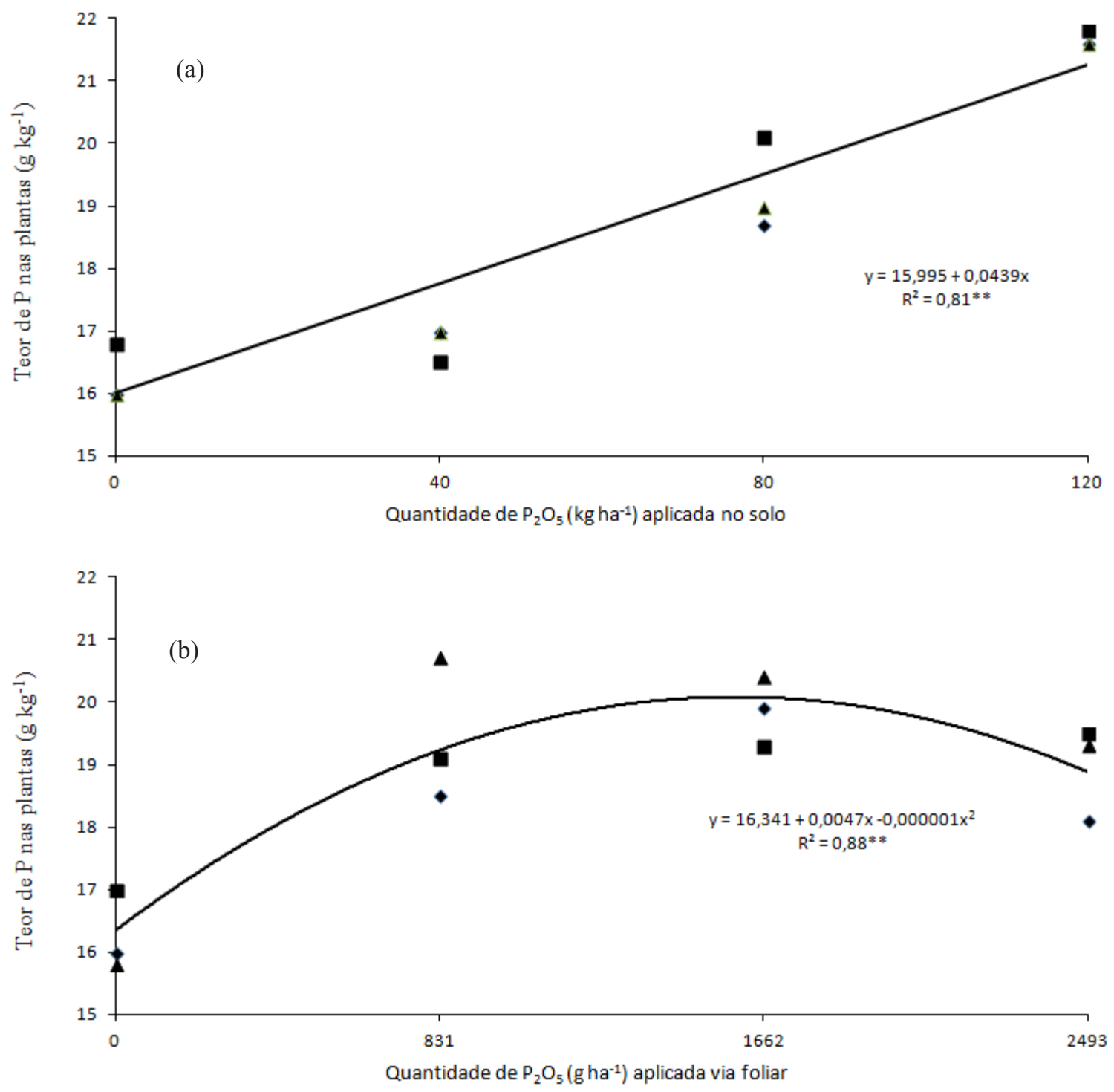

Fonte: Elaborado pelos autores. 
Desta forma, é necessário garantir o suprimento contínuo de P até a fase de maturação, a fim de que não haja redução na produção dos grãos (PETRILLI, 2007). Neste sentido, verificou-se a importância da adubação foliar, visando complementar o $P$ fornecido via solo. Vale destacar, que a resposta não é linear, e a aplicação de doses superiores a $1662 \mathrm{~g} \mathrm{ha}^{-1}$ de $\mathrm{P}_{2} \mathrm{O}_{5}$ não proporciona incrementos na produtividade, além de aumentar os custos de produção. De acordo com Haq e Mallarino (2000) e Noack, McBeath e McLaughlin (2010), a eficiência do uso da aplicação foliar é dependente da quantidade de P no solo, espécie utilizada, formulação do fertilizante e condições climáticas. Assim com os resultados obtidos em três safras agrícolas, pode-se inferir que a utilização da adubação foliar se mostrou prática viável, proporcionando incrementos significativos na produtividade de grãos do feijoeiro.

Concluí-se com esse trabalho que: o aumento das doses de $\mathrm{P}$ aplicadas via solo e foliar proporcionaram aumento do número vagens $\mathrm{m}^{-2}$, produtividade e teor de $\mathrm{P}$ na planta do feijoeiro comum;a maior produtividade do feijoeiro $\left(3.446 \mathrm{~kg} \mathrm{ha}^{-1}\right)$ foi obtida com a combinação de $120 \mathrm{~kg} \mathrm{ha}^{-1}$ de $\mathrm{P}_{2} \mathrm{O}_{5}$ via solo com 1.662 g de $\mathrm{P}_{2} \mathrm{O}_{5}$ ha $^{-1}$ via foliar; e a resposta da cultura à aplicação de $\mathrm{P}$ no solo é incrementada com a aplicação do fósforo via foliar.

\section{Referências}

ALVARENGA, M. A. R.; SILVA, E. C.; SOUZA, R. J.; CARVALHO, J. G. Efeito de doses de nitrogênio aplicadas no solo e níveis de cálcio aplicados via foliar sobre o teor e o acúmulo de micronutrientes em alface americana. Ciência e Agrotecnologia, Lavras, v. 24, n. 4, p. 905-916, out./dez. 2000.

ANDRADE, C. A. B.; PATRONI, S. M. S.; CLEMENTE, E.; SCAPIM, C. A. Produtividade e qualidade nutricional de cultivares de feijão em diferentes adubações. Ciência e Agrotecnologia, Lavras, v. 28, n.5, p. 1077-1086. 2004.

ARF, M. V.; BUZETTI, S.; ARF, O.; KAPPES, C.; FERREIRA, J. P.; GITTI, D. C.; YAMAMOTO, G. J. T. Fontes e épocas de aplicação de nitrogênio em feijoeiro de inverno sob sistema plantio direto. Pesquisa Agropecuária Tropical, Goiânia, v. 41, n. 3, p. 430-438. 2011.

BORKERT, C. M. Soja: adubação foliar. Londrina: EMBRAPA-CNPSo, 1987. 34 p. (Embrapa Soja. Documentos, 22).

ClAESSEN, M. E. C. (Org.). Manual de métodos de análise de solo. 2. ed. Rio de Janeiro: EMBRAPA-CNPS, 1997. 212 p. (Documento 1).

CONAB. Acompanhamento de safra brasileira: grãos, décimo segundo levantamento, setembro 2013 / Companhia Nacional de Abastecimento. Brasília: Conab, 2011. Disponível em: <http://www.conab.gov. br/OlalaCMS/uploads/arquivos/13_10_16_14_32_01_ boletim_portugues_-_setembro_2013.pdf $>$. Acesso em: 22 out. 2013.

DIXON, R. C. Foliar fertilization improves nutrient use efficiency. Fluid Journal, New York, v. 11, p. 22-23, 2003.

DOORENBOS, J.; PRUITT, W. O. Crop and water requirements. Roma: FAO, 1976. 179 p. (FAO Irrigation and Drainage Paper n. 24).

EMPRESA BRASILEIRA DE PESQUISA AGROPECUÁRIA - EMBRAPA. Sistema brasileiro de classificação de solos. Brasília: EMBRAPA-CNPS, 1999. $412 \mathrm{p}$.

FAGERIA, N. K.; BALIGAR, V. C. Response of lowland rice and common bean grown in rotation to soil fertility levels on a varzea soil. Fertilizer Research, Dordrecht, v. 45, n. 1, p. 13-20, 1996.

FAGERIA, N. K.; BARBOSA FILHO, M. P.; STONE, L. F. Nutrição de fósforo na produção do feijoeiro. In: SIMPÓSIO SOBRE FÓSFORO NA AGRICULTURA BRASILEIRA, 2004, Piracicaba. Anais... Piracicaba: POTAFOS, 2004. p. 435-455.

FAGERIA, N. K.; SANTOS, A. B.; MOREIRA, A. Yield, nutrient uptake and changes in soil chemical properties as influenced by liming and iron application in common bean in a no-tillage system. Communications in Soil Science and Plant Analysis, Georgia, v. 41, n. 14, p. 1740-1749, 2010.

GIRMA, K.; MARTIN, L.; FREEMAN, K.; MOSALI, J.; TEAL, R.; RAUN, W. R.; MOGES, S.; ARNALL, D. Determination of optimum rate and growth stage for foliar-applied phosphorus in corn. Communications in Soil Science and Plant Analysis, Georgia, v. 38, n. 9-10, p. 1137-1154, 2007. 
HAQ, M. U.; MALLARINO, A. P. Soybean yield and nutrient composition as affected by early season foliar fertilization. Agronomy Journal, Madison, v. 92, n. 1, p. 16-24, 2000.

HUMBERT, R. P. The Growing of sugar cane. 3. ed. New York: Elsevier, 1983. 128 p.

KUBOTA, F. Y.; ANDRADE NETO, A. C.; ARAUJO, A. P.; TEIXEIRA, M. G. Crescimento e acumulação de nitrogênio de plantas de feijoeiro originadas de sementes com alto teor de molibdênio. Revista Brasileira de Ciência do Solo, Viçosa, v. 32, n. 4, p. 1635-1641, 2008.

MALAVOLTA, E. Elementos de nutrição mineral de plantas. Piracicaba: Ceres, 1980. 215 p.

MELÉM JÚNIOR, N. J.; BRITO, O. R.; FONSCECA JÚNIOR, N. S.; FONSCECA, I. C. B.; AGUIAR, S. $\mathrm{X}$. Nutrição mineral e produção de feijão em áreas manejadas com e sem queima de resíduos orgânicos e diferentes tipos de adubação. Semina: Ciências Agrárias, Londrina, v. 32, n. 1, p. 7-18, jan./mar. 2011.

MIRANDA, L. N.; AZEVEDO, J. A.; MIRANDA, J. C. C.; GOMES, A. C. Produtividade do feijoeiro em resposta a adubação fosfatada e a regime de irrigação em solo de Cerrado. Pesquisa Agropecuária Brasileira, Brasília, v. 35, n. 4, p. 703-710, 2000.

MURAOKA, T.; NEPTUNE, A. M. L. Efeito da aplicação foliar de polifosfato, superfosfato, uréia e yogen na produção do feijoeiro (Phaseolus vulgaris L.). In: SIMPÓSIO DE ADUBAÇÃO FOLIAR, 1., 1981, Botucatu. Anais... Botucatu: FEPAF, 1981. p. 120.

NOACK, S. R.; McBEATH, T. M.; McLAUGHLIN, M. J. Potential for foliar phosphorus fertilisation of dryland cereal crops: a review. Crop and Pasture Science, Collingwood, v. 61, n. 8, p. 659-669, 2010.

PELÁ, A.; RODRIGUES, M. S.; SANTANA, J. S.; TEIXEIRA, I. R. Fontes de fósforo para a adubação foliar na cultura do feijoeiro. Scientia Agraria, Curitiba, v. 10, n. 3, p. 313-318, jul./ago. 2009.

PELÁ, A.; ZUCARELLI, C.; SOUTO, L. S.; WADT, L. G. R.; MARTINEZ, M. M.; GRASSI FILHO, H. Aplicação de fósforo via foliar durante o desenvolvimento do feijoeiro. In: CONGRESSO BRASILEIRO DE CIÊNCIA DO SOLO, 29., 2003, Ribeirão Preto. Anais... Ribeirão Preto: [s. n.], 2003. 1 CD-ROM.

PETRILLI, L. R. T. C. Doses e modos de aplicação de fósforo na nutrição e produção do feijoeiro cultivar Pérola. 2007. Dissertação (Mestrado em Agronomia) - Faculdade de Ciências Agronômicas. Universidade Estadual Paulista Julio de Mesquita Filho, Botucatu.
PRIMAVESI, O. Resultados de Nitrofoska foliar em diversas culturas no Brasil. In: SIMPÓSIO DE ADUBAÇÃO FOLIAR, 1., 1980, Botucatu. Anais... Botucatu: FEPAF, 1981. p. 73-109.

REZENDE, P. M.; GRIS, C. F.; CARVALHO, J. G.; GOMES, L. L.; BOTTINO, L. Adubação foliar. I. Épocas de aplicação de fósforo na cultura da soja. Ciência e Agrotecnologia, Lavras, v. 29, n. 6, p. 1105-1111, nov./ dez. 2005.

ROSOlEM, C. A.; BOARETTO, A. E. Adubação foliar do feijoeiro. In: SIMPÓSIO BRASILEIRO DE ADUBAÇÃO FOLIAR, 2., Botucatu, 1987. Anais... Campinas: Fundação Cargill, 1987. p. 449-512.

SILBERSTEIN, O.; WITTWER, S. H. Foliar application of phosphatic nutrients to vegetable crops. Journal of the American Society for Horticultural Science, Alexandria, v. 58, n. x, p. 179-190, 1951.

SILVA, P. R. C. Processo de produção de adubos foliares. Salvador: Serviço Brasileiro de Respostas Técnicas, Rede de Tecnologia da Bahia, 2006. 5 p.

SILVA, S. C. DA; HEINEMANN, A. B.; PAZ, R. L. F.; AMORIM, A. DE O. Informações meteorológicas para pesquisa e planejamento agrícola, referentes ao ano de 2009, do município de Santo Antônio de Goiás, GO. Santo Antônio de Goiás: Embrapa Arroz e Feijão, 2010. 32 p. (EMBRAPA, CNPAF. Documentos, 256).

VALDERRAMA, M.; BUZETTI, S.; BENETT, C.G.S.; ANDREOTTI, M.; ARF, O.; SÁ, M. E. Fontes e doses de nitrogênio e fósforo em feijoeiro no sistema plantio direto. Pesquisa Agropecuária Tropical, Goiânia, v. 39, n. 3, p. 191-196, 2009.

VIEIRA, C. Adubação mineral e calagem. In: VIEIRA, C.; PAULA JÚNIOR, T. J.; BORÉM, A. (Ed.). Feijão. 2. ed. Viçosa: UFV, 2006. p. 115-142.

ZUCARELI, C.; PRANDO, A. M.; RAMOS JUNIOR, E. U.; NAKAGAWA, J. Fósforo na produtividade e qualidade de sementes de feijão Carioca Precoce cultivado no período das águas. Revista Ciência Agronômica, Fortaleza, v. 42, n. 1, p. 32-38, 2011.

ZUCARELI, C.; RAMOS JUNIOR, E. U.; BARREIRO, A. P.; NAKAGAWA, J.; CAVARIANI, C. Adubação fosfatada, componentes de produção, produtividade e qualidade fisiológica em sementes de feijão. Revista Brasileira de Sementes, Londrina, v. 28, n. 1, p. 9-15, 2006.

ZUCARELI, C.; RAMOS JUNIOR, E. U.; OLIVEIRA, M. A.; CAVARIANI, C.; NAKAGAWA, J. Índices biométricos e fisiológicos em feijoeiro sob diferentes doses de adubação fosfatada. Semina: Ciências Agrárias, Londrina, v. 31, p. 1313-1324, 2010. Suplemento 1. 